\title{
The impact of chronic intermittent hypoxia on the expression of intercellular cell adhesion molecule-1 and vascular endothelial growth factor in the ischemia-reperfusion rat model
}

\author{
Meili Yang ${ }^{1}$, Yafang Chen ${ }^{1}$, Zhengang $\mathrm{Wu}^{2}$, Yaping Zhang ${ }^{3}$, Ruowei Cai ${ }^{1}$, Lichao Ye ${ }^{1}$, Yinhui Huang ${ }^{4}$, \\ Lingxing Wang ${ }^{1}$, Huiling $\mathrm{He}^{1}$ \\ ${ }^{1}$ Department of Neurology, the Second Affiliated Hospital of Fujian Medical University, Quanzhou 362000, Fujian Province, \\ 2Department of Surgery, Quanzhou Medical College, Quanzhou 362000, Fujian Province, ${ }^{3}$ Department of Scientific Research Center, \\ the Second Affiliated Hospital of Fujian Medical University, Quanzhou 362000, Fujian Province, ${ }^{4}$ Department of Neurology, Jinjiang \\ Hospital, Jinjiang 362200, Fujian Province, China
}

\begin{abstract}
The aim of this study was to investigate the effect and mechanism of chronic intermittent hypoxia $(\mathrm{CIH})$ on cerebral injury using the ischemia-reperfusion rat model. In total, 36 SD rats were divided into three groups: pseudo-surgery group (sham group), ischemia-reperfusion (IR) group (CIR group), and $\mathrm{CIH}$ intervention group (CIH group). The IR model was established using the suture-occluded method. $\mathrm{CIH}$ intervention was performed starting at 12 weeks prior to the establishment of the IR model for rats in the $\mathrm{CIH}$ group. Ultra-microstructure was examined using an electron microscope. Expression of intercellular cell adhesion molecule-1 (ICAM-1) and vascular endothelial growth factor (VEGF) in rat brain tissue was evaluated by immunohistochemical methods and western blot assays. The neurological deficit scores of rats in the $\mathrm{CIH}$ group were higher than those in the CIR group $(p<0.05)$. Using electron microscopy, we observed more severe edema around the capillaries of the rat brain that resulted in more pressure on the vascular wall of the capillaries in the CIH group compared to the CIR group. The expression of ICAM-1 and VEGF in rat brain tissue was rare in the sham group, but was significantly elevated in the CIR group $(p<0.05)$ and even higher in the CIH group, compared to the CIR group. Hence, the brain injury in ischemia-reperfusion rat models following $\mathrm{CIH}$ intervention may be related to the increased expression of ICAM-1 and VEGF.
\end{abstract}

Key words: chronic intermittent hypoxia, ischemic reperfusion injury, ICAM-1, VEGF.

\section{Introduction}

Obstructive sleep apnea (OSA) and stroke are common and closely related diseases [18]. OSA was found in about one-half to three-quarters of stroke patients, according to a report by the American Heart Association/American Stroke Association of
2014 [16]. Furthermore, an epidemiologic study has demonstrated that OSA was an independent risk factor for cerebrovascular diseases, which affects the prognosis of stroke [21] and increases the incidence of cardiovascular and cerebrovascular events in stroke patients [11]. The molecular mechanisms

\section{Communicating author}

Lingxing Wang, Department of Neurology, the Second Affiliated Hospital of Fujian Medical University, No. 34 Zhongshan North Road, Quanzhou 362000, Fujian Province, China, phone: +86 595 22788097, fax: +86 595 22770258, e-mail: cnlingxingwang@163.com 
behind the induction and exacerbation of cerebrovascular disease by OSA remain ambiguous. Possible theories behind the pathogenesis of OSA in cerebrovascular disease include sympathetic activation, inflammatory reactions, and endothelial dysfunction, hypercoagulable states, and metabolic disorders induced by oxidative stress $[6,15]$. Researchers have speculated that the repetitive short episodes of hypoxia/reoxygenation in OSA activate inflammatory pathways, further resulting in the activation of inflammatory cells and the expression of inflammatory cytokines $[7,20]$. OSA was also found to induce oxidative stress responses and lipid peroxidation, leading to vascular endothelial cell function dysfunction, and further leading to atherosclerosis $[23,27]$. Presently, most reports regarding the relationship between OSA and stroke are based on the clinical observation with few cellular assays, intact animal studies, and prospective studies. Furthermore, research regarding the link between chronic intermittent hypoxia $(\mathrm{ClH})$ and inflammation in the brain following ischemia-reperfusion are also rare. In this study, $\mathrm{ClH}$ intervention was performed to evaluate the expression of intercellular adhesion molecule 1 (ICAM-1) and vascular endothelial growth factor (VEGF), and to investigate the effect of $\mathrm{CIH}$ on inflammatory vascular injury after ischemia-reperfusion injury, providing a potential therapeutic target against OSA combined with a stroke.

\section{Material and methods \\ Animal grouping}

Thirty-six male Sprague-Dawley rats (Shanghai SLAC Laboratory Animal Co., Ltd, Shanghai, China) with the mean weight of $250 \pm 30 \mathrm{~g}$, were randomly divided into three groups according to the random number table: 12 rats for the pseudo-surgery group (sham group), 12 for the suture-occluded group (CIR group), and 12 for the chronic intermittent hypoxia group (ClH group). In all three groups, half of the rats were examined by the western blot assay and electron microscopy, and the other half by the IHC assay. This study was carried out in strict accordance with the recommendations in the Guide for the Care and Use of Laboratory Animals of the National Institutes of Health. The animal use protocol has been reviewed and approved by the Institutional Animal Care and Use Committee (IACUC) of Fujian Medical University.

\section{Rat model establishment}

$\mathrm{CIH}$ intervention was performed for the rats in the $\mathrm{CIH}$ group for 12 weeks: a sealed Plexiglas box was fabricated as previously described [19], where the oxygen concentration within the box was adjusted by regulating the type and amount of air inflow. The various gases were introduced in the following order for each 4-minute intermittent hypoxia cycle: nitrogen for $120 \mathrm{~s}, 30 \mathrm{~s}$ rest, oxygen for $60 \mathrm{~s}$, and air for $30 \mathrm{~s}$. During the cycle, the maximum and minimum oxygen concentrations were $23.3 \pm 0.47 \%$ and $8.3 \pm 0.31 \%$, respectively. Rats in the $\mathrm{ClH}$ group were placed in the box to undergo intermittent hypoxia from $8 \mathrm{am}$ to $4 \mathrm{pm}$, and then placed in an atmospheric environment after 4 pm each day for 12 consecutive weeks. Rats in the remaining two groups were fed in the same environment and with the same conditions, with no $\mathrm{ClH}$ intervention.

The rat ischemia-reperfusion injury (IRI) model was established as follows: a nylon thread with a diameter of $0.235 \mathrm{~mm}$ and a length of $5 \mathrm{~cm}$ was taken as the bolting line, and its head end was encased with paraffin so that the diameter of its head end was $0.25-0.30 \mathrm{~mm}$. Middle cerebral artery occlusion (MCAO) was performed on the rats in CIR and $\mathrm{CIH}$ groups, according to the method by Longa et al. [14]. Following the intraperitoneal injection of the anesthetic, the rat was fixed on the operating table, exposing the right common carotid artery and the vagus nerve. The blunt separation of the internal carotid artery and external carotid artery was performed along a thin line in the right common carotid artery deep surface and the branches of the external carotid artery on the thyroid artery and the occipital artery were burned out to expose around 3-4 $\mathrm{mm}$ of the external carotid artery. In the deep surface, where two thin lines (near the side of the slipknot, into the root of the external carotid artery, external carotid artery distal ligation), separate the internal carotid artery and its deep branch, and in its bifurcation ligation wing palatine artery, the common carotid artery and internal carotid artery were clipped. On the external carotid artery, a "V" type of incision was performed and the head end of the nylon thread was inserted into the common carotid artery and the external carotid artery to the below one to join them in a straight line. The plug wire was inserted via common carotid artery bifurcation into the internal carotid artery, and the plug 
line was slowly inserted along the internal carotid, proximal, and middle cerebral arteries. The depth of the plug wire from the outside of the internal carotid artery bifurcation point should be $18 \pm 0.5 \mathrm{~mm}$ in order to block the arterial blood supply to the side of the brain. The root nodule of the external carotid artery was ligated and the common carotid artery clamp was loosened. After the skin was sutured, the wire was placed outside with a total length of about $5 \mathrm{~cm}$. In the sham group, the surgical procedures were identical to those in the ischemic group, except that the suture line was inserted into the artery of the rats with a depth less than $10 \mathrm{~mm}$. The reperfusion model was established by pulling out all of the suture lines after two hours of ischemia in the artery of the rat brain. At $24 \mathrm{~h}$ after the establishment the model, neurological deficit scores of rats were rated on a five-point scale according to the research of Longa et al. [14]. The rats were euthanized following the neurological assessment.

\section{Neurological deficit scoring}

Unsuccessful models, including rats with the scores of 0 or 4 , rats with qualified scores but with subarachnoid hemorrhage, and rats that died during or after surgery were excluded.

\section{Electron microscope examination}

The rat brain was rapidly excised and ultrathin sections were prepared from a one cubic milliliter block of tissue from the left cortices at the crossing point at $4 \mathrm{~mm}$ behind the frontal pole and $4 \mathrm{~mm}$ left of the midline. The sections were double stained with uranyl acetate and lead citrate and examined with a H7650 TEM for changes in the ultra-microstructure of microvessels in the cerebral cortex. The remaining brain tissue was immediately placed in liquid nitrogen to be preserved for further investigation of molecular indicators. Neurons, glial cells, and cerebral blood vessels were photographed under high and low power fields.

\section{Western blot}

After TEM examination, $30 \mathrm{mg}$ of brain tissue surrounding the occlusion in the injured left cortex was removed and homogenized. SDS-PAGE was performed to isolate the protein. The protein was then transferred from the gel to a PVDF membrane. After two hours of blocking at room temperature $\left(-25^{\circ} \mathrm{C}\right)$ using 5\% skimmed milk powder, the membranes were incubated with the primary antibody (rabbit anti-rat ICAM-1, rabbit anti-rat VEGF, respectively) (Lebing ying Biotechnology, Fuzhou, China) on a $4^{\circ} \mathrm{C}$ shaker overnight. After washing the membranes with TBS-T, they were then incubated with the secondary antibody for one hour. Finally, the membranes were washed thoroughly again with TBS-T and photographed. The film was developed and fixed using the ECL system and the expression levels of ICAM-1 and VEGF were evaluated. The internal reference was $\beta$-actin.

\section{IHC assay}

All the rats were handled at the same time. The brains were isolated and the expression status of ICAM-1 and VEGF (Santa Cruz Biotechnology, Inc., Santa Cruz, USA) was evaluated by the MaxVision ${ }^{\mathrm{TM}}$ method (Fuzhou Maixin Biological Technology Co., Ltd., Fuzhou, China). Positive expression of ICAM-1 was indicated by the brown-stained particle deposition within or surrounding the microvessel walls. Five non-overlapping views using a 400× light microscope and the same background light strength were randomly selected to count the number of blood vessels with a positive ICAM-1 expression. The total number of five views was considered to be the number of blood vessels for each slice. VEGF expression was revealed as yellow or brown particle deposition in the cytoplasm of cells. Slices were prepared using brain tissue isolated from the right prefrontal cortex, and five non-overlapping views using a 400x light microscope were randomly selected from these slices and photographed. The photos were analyzed using Image-Pro Plus 6.0, and the integral optical density (IOD) of the positive area was measured to reflect the total amount of protein expression. The mean value of the five views was considered to be the content of VEGF for each slice and used for statistical analysis. A randomized controlled trial was carried out to read the slices, and the results of this study were re-examined by pathological researchers in our hospital.

\section{Statistical analysis}

Data were analyzed using SPSS 16.0, and all the data were represented using $\bar{x} \pm s$. The difference of means among multiple groups was compared using one-way ANOVA and difference of means between 
two groups was compared using $t$-test. $P \leq 0.05$ was considered statistically significant.

\section{Results}

\section{Comparison of neurological deficit scores}

The neurological score of rats in the sham group was zero, while different levels of focal nervous functional disorders were observed for the rats in $\mathrm{CIR}$ and $\mathrm{CIH}$ groups after ischemia-reperfusion injury, such as left lower limb weakness, circling toward the left side, falling down, and disturbance of consciousness. The difference between the scores of the $\mathrm{CIR}$ and $\mathrm{CIH}$ groups was statistically significant $(t=3.404, p<0.01$, Table I).

\section{TEM examination}

The structure of the micro-vessels within the brain cortex of rats in the sham group was intact, with no edema in the basal lamina or surrounding vascular wall. The vascular basal lamina was also continuously intact. The structure of mitochondria was clear, and the rough endoplasmic reticulum (ER) was normal. Several microvilli were found at the

Table I. The neurologic deficit scores of rats in different groups after IRI

\begin{tabular}{|lc|}
\hline Groups & Neurologic deficit score \\
\hline Sham & 0 \\
\hline $\mathrm{CIR}$ & $1.72 \pm 0.68^{\Delta}$ \\
\hline $\mathrm{CIH}$ & $2.54 \pm 0.59^{\star}$ \\
\hline
\end{tabular}

${ }^{\Delta}$ Compared with that of the sham group $p<0.01$; ${ }^{*}$ Compared with that of the CIR group $p<0.01$ surface of endothelial cells, extruding towards the vascular lumen (Fig. 1A). In the samples from the CIR group, mild edema was observed surrounding the capillaries, and the capillaries were slightly constricted with less microvilli. Mitochondrial edema and degranulation of the rough ER were observed in endothelial cells. Partial edema was also found in the basal lamina. A basement membrane defect in blood vessels was observed (Fig. 1B). In the samples from the $\mathrm{CIH}$ group, severe edema was observed surrounding the capillaries, and the capillaries were significantly constricted with less microvilli present. Fusion of the cristae and the mitochondrial membrane and degranulation of rough ER were observed in endothelial cells. Rupture and damage was also found in the basal lamina (Fig. 1C).

\section{Expression level of ICAM-1 and VEGF protein}

The results of the western blots demonstrated that very little ICAM-1 and VEGF were expressed in cells from the sham group. However, ICAM-1 expression was enhanced in the CIR group, and became even more distinct in the $\mathrm{CIH}$ group. The difference in ICAM-1 expression between the CIR and $\mathrm{CIH}$ groups was statistically significant $(t=2.322, p<0.05$; $t=2.638, p<0.05)$ (Fig. 2, Table II).

\section{Expression of ICAM-1}

Positive reaction of ICAM-1 was indicated by the brown-stained particle deposition within or surrounding the microvessel walls. Rare positive vessels were observed in tissues from the sham group and in the non-ischemic cerebral cortex and hippocampal
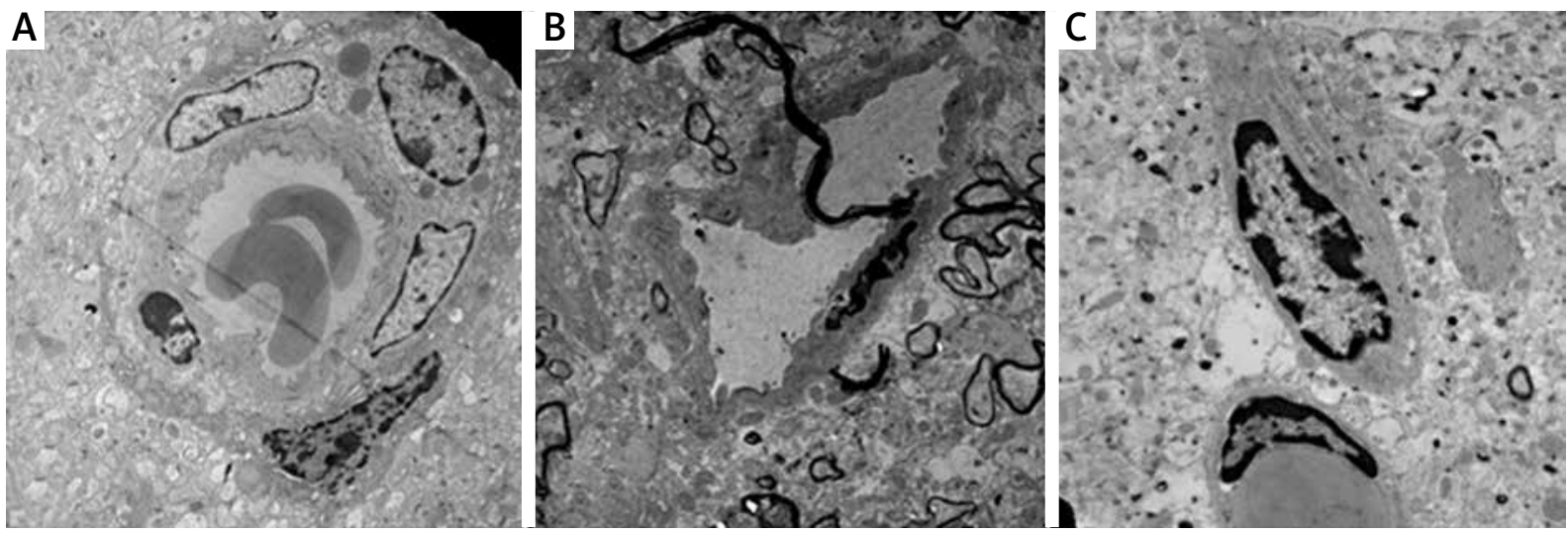

Fig. 1. Examination of vascular damage using TEM. A) Sham group; B) CIR group; C) CIH group. 
area in the CIR group (Fig. 3A). An increased number of positive vessels was observed on the ischemic side of the brain in the CIR group, mostly in the prefrontal cortex and the basal ganglia regions. Expression of ICAM-1 in the cytoplasm of neurons in the peripheral area of necrosis was also observed (Fig. 3B). Brown particle deposition within or surrounding the walls of microvessels was found in the samples of the $\mathrm{CIH}$ group. The number of positive vessels in the $\mathrm{CIH}$ group was significantly larger than that of the CIR group $(t=9.185, p<0.01)$ (Fig. 3C, Table III).

\section{Expression of VEGF}

VEGF expression was revealed as yellow or brown particle deposition in the cytoplasm of cells. Weak VEGF expression was found in the prefrontal cortex area and the corpus striatum of rats in the sham group (Fig. 4A). An increased expression was observed in cells in the CIR group, and the cytoplasm and the nucleus of these cells showed brown staining (Fig. 4B). A strong positive expression was observed in the prefrontal cortex area and the corpus striatum of rats in the $\mathrm{CIH}$ group, with an increased number of positive cells and darker staining (Fig. 4C). The difference compared to that in the CIR group was statistically significant $(t=6.449, p<0.01)$ (Table III).

\section{Discussion}

OSA can result in damage to many organs of the human body, especially the nervous system [1]. Clinical studies have demonstrated that OSA leads to alterations of cerebral hemodynamics, brain metabolism, inflammatory responses, and oxidative stress, as well as the activation and aggravation of cerebral infarctions [4]. In our study, we found that

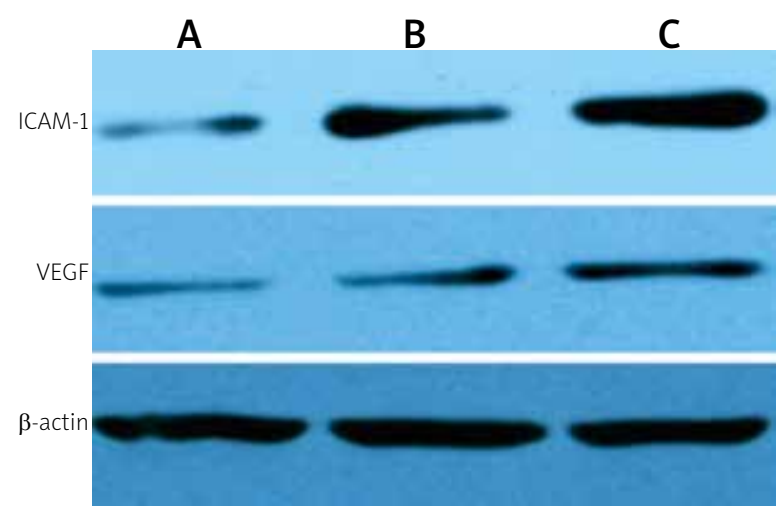

Fig. 2. Western blot results indicating the expression of ICAM-1, VEGF and the internal reference. A) Sham group; B) CIR group; C) $\mathrm{CIH}$ group.

Table II. Expression levels of ICAM-1, VEGF, and $\beta$-actin indicated by the western blot assay

\begin{tabular}{|llcc|}
\hline Groups & $n$ & ICAM-1 & VEGF \\
\hline Sham & 6 & $0.125 \pm 0.019$ & $0.101 \pm 0.023$ \\
\hline CIR & 6 & $0.513 \pm 0.089^{\Delta}$ & $0.194 \pm 0.098^{\Delta}$ \\
\hline $\mathrm{CIH}$ & 6 & $0.586 \pm 0.077^{\star}$ & $0.264 \pm 0.065^{\star}$ \\
\hline
\end{tabular}

${ }^{\Delta}$ Compared with that of the sham group $p<0.01$; ${ }^{*}$ Compared with that of the CIR group $p<0.05$.

Table III. The number of ICAM-1 positive vessels and VEGF expression in different groups

\begin{tabular}{|llcc|}
\hline Groups & $n$ & $\begin{array}{c}\text { Number of ICAM-1 } \\
\text { positive vessel (IOD) }\end{array}$ & VEGF \\
\hline Sham & 6 & 0 & $3674.56 \pm 120.46$ \\
\hline $\mathrm{CIR}$ & 6 & $17.85 \pm 3.78^{\Delta}$ & $9785.64 \pm 857.33^{\Delta}$ \\
\hline $\mathrm{CIH}$ & 6 & $33.45 \pm 4.16^{\star}$ & $13496.22 \pm 1409.36^{\star}$ \\
\hline
\end{tabular}

${ }^{\Delta}$ Compared with that of the sham group $p<0.05$; ${ }^{*}$ Compared with that of the CIR group $p<0.01$.
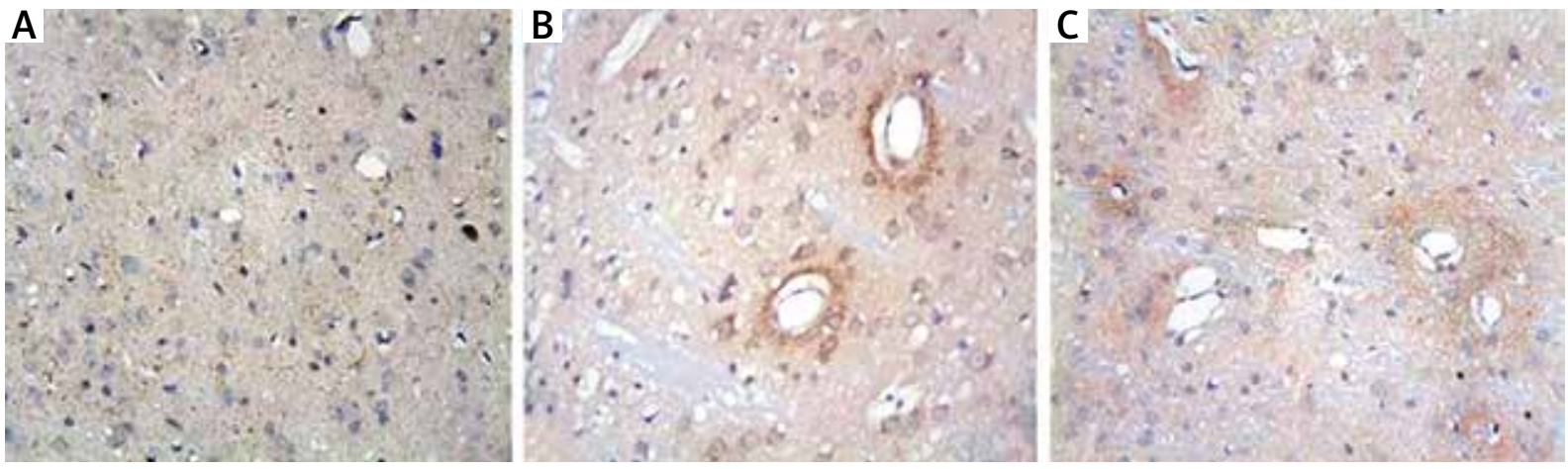

Fig. 3. IHC assay demonstrating the expression of ICAM-1. A) Sham group; B) CIR group; C) CIH group. 

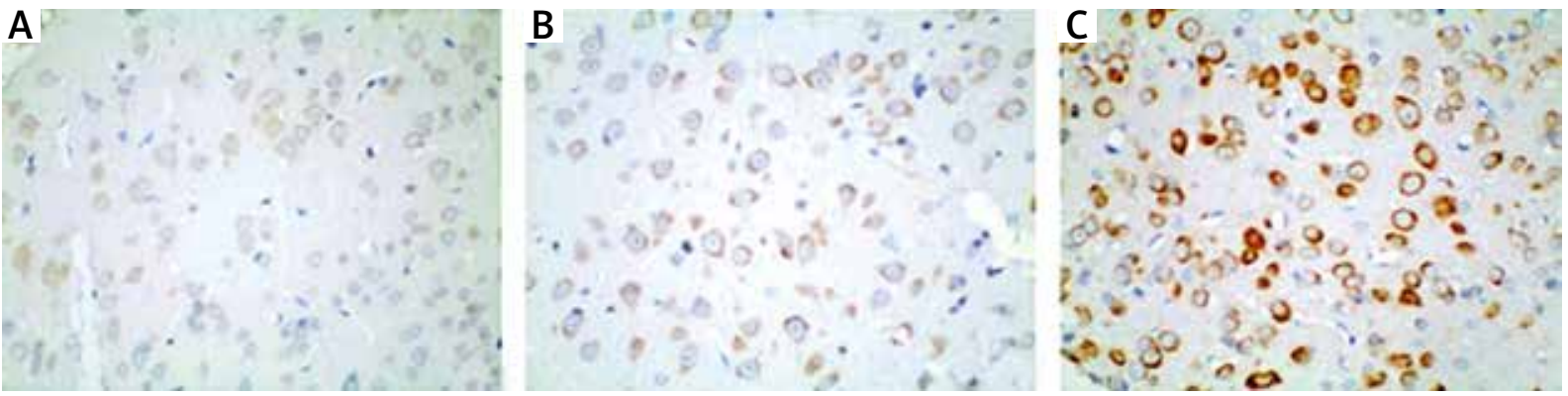

Fig. 4. IHC assay demonstrating the expression of VEGF. A) Sham group; B) CIR group; C) CIH group.

the neurological deficit scores of rats after 12 weeks of $\mathrm{CIH}$ using the suture-occluded method was significantly increased compared to rats that received ischemia-reperfusion only. This observation is in agreement with clinical data that indicates that $\mathrm{CIH}$ exacerbates damage to the rat nervous system after ischemia-reperfusion and that $\mathrm{ClH}$ was one factor leading to the poor prognosis of cerebral ischemia-reperfusion.

Vasogenic cerebral edema was induced by a series of damage factors generated at different times after cerebral ischemia-reperfusion. Constriction of microvessels, damaged to vascular endothelial cells, and activation of the coagulation mechanism further blocks these vessels, finally leading to aggravated neurological damage [9]. One report has demonstrated that intermittent hypoxemia and over-excited sympathetic nerves during night stimulated oxidative stress can enhance the expression of adherence molecules and pro-inflammatory factors, induce systematic inflammatory responses, and damage endothelial functions leading to atherosclerosis in OSA patients [3]. Meanwhile, another study has indicated that in patients with OSA, the blood viscosity is increased, blood speed is decreased, and blood platelet accumulation is accelerated, facilitating thrombosis, vascular stenosis, and occlusion [22]. We examined the ultra-microstructure of brain tissues and demonstrated that after cerebral ischemia-reperfusion, mild edema surrounding the capillaries was observed and the capillaries were slightly constricted. Mitochondrial edema and degranulation of rough ER were observed in endothelial cells. Partial distortion and edema was also found in the basal lamina. Additionally, a basement membrane defect of blood vessels was observed and vasogenic and cytotoxic brain edema appeared. In samples from the $\mathrm{ClH}$ group, more severe injuries were observed, which further indicated that $\mathrm{ClH}$ exacerbates neurological damage by aggravating the vascular endothelial injury of capillaries after ischemia-reperfusion. Another study has shown that the ultrastructure of glial cells was swollen and degenerated, as shown by electron microscopy, following cerebral ischemia reperfusion in rats [26]. Studies have also found that astrocyte activity is increased, resulting in glial cell hyperplasia and hypertrophy, increased collagen fiber acidic protein expression levels, and the production of a variety of inflammatory factors, leading to brain tissue damage $[8,25]$. However, few studies have been carried out regarding the effect of chronic intermittent hypoxia on glial cells in rats. It is regrettable that this study has not been given sufficient attention and research.

$\mathrm{CIH}$ has been proven to induce vascular inflammatory responses and promote the pathogenesis of atherosclerosis, however, its exact mechanisms are unknown. One study has speculated that this process may result from oxidative stress and vascular endothelial function disorders caused by the modulated transcription and expression of adherence molecules [13]. ICAM-1, also named as CD54, is a single chain transmembrane glycoprotein belonging to the immune globulin superfamily, and is widely distributed throughout the body. It is considered to be one of the markers of endothelial cell activation. Under normal physiological conditions, ICAM-1 is poorly expressed or not expressed at all. Cerebral ischemia has been demonstrated to stimulate leukocytes and brain vascular endothelial cells, leading to the enhanced synthesis of adhesion molecules associated with inflammation, including ICAM-1 and vascular cell adhesion molecules 1 (VCAM-1), and promote the infiltration of leukocytes to the cerebral infarction area. Reperfusion further exacerbates the leukocyte migration to the infarction area resulting in 
the aggravated inflammatory injury [5]. Arnaud et al. found that intermittent hypoxia can induce global inflammatory responses and facilitate the expression of ICAM-1 in mesentery vessels in C57BL6 mice after intermittent hypoxia, suggesting that intermittent hypoxia induces the vascular injury at least in part, through the production of inflammatory factors [2]. A rare positive ICAM-1 expression around vessels indicated by $\mathrm{IHC}$ and western blot assays was observed in tissues from the sham group. This expression was only occasionally found in the choroid plexus and the cytoplasm of a few neurons. More positive vessels were observed in rats from the CIR group, mostly in the ischemic side of the brain and the peripheral area, as well as the cytoplasm of neurons in the necrotic area. These results confirm that the increased expression of ICAM-1 after ischemia-reperfusion leads to the induction of contact and adhesion between leukocytes and endothelium. This is one of the core pathological symptoms of inflammation, and aggravation of tissue edema and secondary injury, consistent with a large number of animal studies worldwide [24]. By counting the number of positive vessels after immune staining, ICAM-1 expression was found to be further increased in samples from the $\mathrm{ClH}$ group, indicating that $\mathrm{CIH}$ may exacerbate the inflammatory injury of cerebral ischemia by promoting the expression of ICAM-1.

VEGF is a multi-functional cytokine. VEGF expression increases following cerebral ischemia and promotes angiogenesis in cooperation with many other angiogenic growth factors, exerting a neuroprotective effect. However, VEGF also enhances vascular permeability, the critical cause of vasogenic cerebral edema, through the binding of VEGF and VEGF2 receptor and acting on vascular endothelium through the P13K/AKT signaling pathway [10]. VEGF also increases the permeability of vascular endothelial cells to low density lipoprotein cholesterol (LDL-C), stimulating the high expression of adherence factors, and promoting the pathogenesis of atherosclerosis [17]. In the IHC and western blot assays of our study, poor VEGF expression was found in the cortex and hippocampus of rats in the sham group, while enhanced VEGF expression was observed in the tissues of the CIR group, indicating the involvement of abnormal VEGF expression in cerebral IRI. A previous study has demonstrated that a severe endothelial cell dysfunction and increased expression of markers of endothelial cell damage, including VEGF and ICAM-1, is observed in the circulation of OSA patients [28]. Another report demonstrated that the secretion of VEGF was facilitated by increased levels of HIF-1a due to the aggravated hypoxia of OSA patients, resulting in endotheliosis, augmented permeability of microvessels, and increased inflammatory responses, further resulting in a tremendously increased incidence of cardiac-cerebral vascular disease [12]. Our study has demonstrated that the expression of VEGF is increased remarkably in the brain cortex of $\mathrm{ClH}$-intervened cerebral ischemia-reperfusion rats, indicating a higher level of inflammatory responses. Therefore, we speculate that $\mathrm{CIH}$ may participate in the pathogenesis of cerebral vascular injury in sleep apnea syndrome (SAS) by promoting the expression of VEGF.

In our study, the focal IRI rat model combined with $\mathrm{CIH}$ intervention was established. The results of neurological deficit scoring, TEM examination, $\mathrm{IHC}$ and western blot assays suggest that aggravated IRI due to $\mathrm{ClH}$ may be related to the increased expression of ICAM-1 and VEGF, indicating that OSA induces inflammation and exacerbates the stroke. This study provides new potential therapeutic targets against OSA combined with the stroke. Further studies are still required, as the rat model cannot completely mimic the pathogenesis of human OSA, and the number of rat models are currently limited.

\section{Acknowledgements}

This study was supported by the scientific program for young scientists of the Health and Family Planning Commission, Fujian Province (2014-1-65), nursery scientific program for young researchers of Fujian Medical University (2014MP032), educational and scientific program for young and middle-age teachers of the Education Department of Fujian Province (JAT160193), and scientific program for elite researchers of the Second Affiliated Hospital of Fujian Medical University (2016MP08).

\section{Disclosure}

The authors declare no conflict of interest.

\section{References}

1. Aaronson JA, van Bennekom CA, Hofman WF, van Bezeij T, van den Aardweg JG, Groet E, Kylstra WA, Schmand B. Obstructive sleep apnea is related to impaired cognitive and functional status after stroke. Sleep 2015; 38: 1431-1437. 
2. Arnaud C, Beguin PC, Lantuejoul S, Pepin JL, Guillermet C, Pelli G, Burger F, Buatois V, Ribuot C, Baguet JP, Mach F, Levy P, Dematteis $M$. The inflammatory preatherosclerotic remodeling induced by intermittent hypoxia is attenuated by RANTES/ CCL5 inhibition. Am J Resipir Crit Care Med 2011; 184: 724-731.

3. Attal P, Chanson P. Endocrinea spects of obstructive sleep apnea. J Clin Endorinol Metab 2010; 95: 483-495.

4. Dyugovskaya L, Lavie P, Lavie L. Increased adhesion molecules expression and production of reactive oxygen species in leukocytes of sleep apnea patients. J Respir Crit Care Med 2002; 165: 934-939.

5. Eltzschig HK, Eckle T. Ischemia and reperfusion from mechanism to translation. Nat Med 2011; 17: 1391-1401.

6. Feng J, Zhang D, Chen B. Endothelial mechanisms of endothelial dysfunction in patients with obstructive sleep apnea. Sleep Breath 2012; 16: 283-294.

7. Guven SF, Turkkani MH, Ciftci B, Ciftci TU, Erdogan Y. The relationship between high sensitivity $C$-reactive protein levels and the severity of obstructive sleep apnea. Sleep Breath 2012; 16: 217-221.

8. Hamanaka J, Hara H. Involvement of Toll-like receptors in ischemia-induced neuronal damage. Cent Nerv Syst Agents Med Chem 2011; 11: 107-113.

9. Hamann GF, del Zoppo GJ. The cerebral microvasculature and responses to ischemia. Stroke 2004; 38: 775-783.

10. Kilic E, Kilic U, Wang Y, Bassetti CL, Marti HH, Hermann DM. Thephosphatidylinositol-3 kinase/Akt pathway mediates VEGF's neuroprotective activity and induces blood brain barrier permeability after focal cerebral ischemia. FASEB J 2006; 20: 1185-1187.

11. King S, Cuellar N. Obstructive sleep apnea as an independent stroke risk factor: A review of the evidence, stroke prevention guidelines, and implications for neuroscience nursing practice. J Neurosci Nurs 2016; 48: 133-142.

12. Kulkas A, Tiihonen P, Eskola K, Julkunen P, Mervaala E, Töyräs J. Novel parameters for evaluating severity of sleep disordered breathing and supporting diagnosis of sleep apnea-hyponea sysdrom. J Med Eng Technol 2013; 37: 135-143.

13. Lavie L. Sleep-disordered breathing and cerebrovascular disease: a mechanistic approach. Neurol Clin 2005; 23: 1059-1075.

14. Longa EZ, Weinstein PR, Carlson S, Cummins R. Reversible middle cerebral artery occlusion without craniectomy in rats. Stroke 1989; 20: 84-91.

15. Mehra R, Bena J, Walia HK. Clarifying the Role of Hypoxia in Obstructive Sleep Apnea as a Potential Promulgator of Atrial Fibrillation in Ischemic Stroke. J Clin Sleep Med 2017; 13: 667. 668.

16. Meschia JF, Bushnell C, Boden-AlbalaB, Braun LT, Bravata DM, Chaturvedi S, Creager MA, Eckel RH, Elkind MS, Fornage M, Goldstein LB, Greenberg SM, Horvath SE, ladecola C, Jauch EC, Moore WS, Wilson JA; American Heart Association Stroke Council; Council on Cardiovascular and Stroke Nursing; Council on Clinical Cardiology; Council on Functional Genomics and Translational Biology; Council on Hypertension. Guidelines for the primary Prevention of stroke: a statement for healthcare professionals from the American Heart Association/American stroke Association. Stroke 2014; 45: 3754-3832.
17. Min S, Huang J, Li J, Qin X, Tang X, Jin F, Chen S, Jiang C, Zou Z, Peng K, Nuruzzaman M, Zhang J, Luo J, Liu S, Luo Z. ZLM-7 exhibits anti-angiogenic effects via impaired endothelial cell function and blockade of VEGF/VEGFR-2 signaling. Oncotarget 2016; 7: 19018-19030.

18. Nava-GuerraL, TranWH,ChalachevaP,LoloyanS, JoshiB, Keens TG, Nayak KS, Davidson Ward SL, Khoo MC. Model-based stability assessment of ventilatory control in overweight adolescents with obstructive sleep apnea during NREM sleep. J Appl Physiol (1985) 2016; 121: 185-197.

19. Neubauer JA. Invited review: Physiological and pathophysiological responses to intermittent hypoxia. J Appl Physiol (1985) 2001; 90: 1593-1599.

20. Ryan S, McNicholas WT. Intermittent hypoxia and activation of inflammatory molecular pathways in OSAS. Arch Physiol Biochem 2008; 114: 261-266.

21. Sahlin C, Sandberg O, Gustafson Y, Bucht G, Carlberg B, Stenlund $\mathrm{H}$, Franklin KA. Obstructive sleep apnea is a risk factor for death in patients with stroke. Arch Intern Med 2008; 168: 297 301.

22. Turmel J, Sériès F, Boulet LP, Poirier P, Tardif JC, Rodés-Cabeau J, Larose E, Bertrand OF. Relationship between atherosclerosis and the sleep apnea syndrome: an intravascular ultrasound study. Int J Cardiol 2009; 132: 203-209.

23. Unnikrishnan D, Jun J, Polotsky V. Inflammation in sleep apnea: an update. Rev Endocr Metab Disord 2015; 16: 25-34.

24. Vemuganti R, Dempsey RJ, Bowen KK. Inhibition of intercellular adhesion molecule-l protein expression by antisense oligonueleotides is neuroprotective after transient middle cerebral artery occlusion in rat. Stroke 2004; 35: 179-184.

25. Voherra A, Meldolesi J. Astrocytes, from brain glue to communication elements the revolution continues. Nat Rev Neurosci 2005; 6: 626-640

26. Wang XS, MIAO L, Yao XM, Zhu XL. The ultrastructural changes of blood-brain barrier after cerebral ischemia reperfusion in rats. Tianiin Med J 2006; 34: 188-189.

27. Xu XM, Yao D, Cai XD, Ding C, Lin QD, Wang LX, Huang XY. Effect of chronic continual-and intermittent hypoxia-induced systemic inflammation on the cardiovascular system in rats. Sleep Breath 2015; 19: 677-684.

28. Zamarrón C, Riveiro A, Gude F. Circulating levels of vascular endothelial markers in obstructive sleep apnoea syndrome. Effects of nasal continuous positive airway pressure. Arch Med Sci 2011; 7: 1023-1028. 